\title{
Integrals of polynomials associated with tableaux and the Garsia-Haiman conjecture
}

\author{
Charles F. Dunkl ${ }^{1}$, Phil Hanlon ${ }^{2}$ \\ 1 Department of Mathematics, University of Virginia, Charlottesville, VA 22903-3199, USA \\ 2 Department of Mathematics, University of Michigan, Ann Arbor, MI 48109-1003, USA \\ (e-mail: hanlon@math.lsa.umich.edu)
}

Received 29 November 1994; in final form 10 December 1996

\section{Introduction}

In a 1983 paper [M1], I. G. Macdonald introduced his well-known "constant term conjectures." These conjectures concern a certain polynomial $\Delta=\Delta(G, k)$ that is indexed by a semisimple Lie algebra $G$ and a positive integer $k$. The polynomial $\Delta$ lives in $\mathbf{Z}[\Phi, q]$, the group ring of the root lattice $\Phi$ of $G$ over $\mathbf{Z}[q]$. A basis for this ring, over $\mathbf{Z}[q]$, is the set of formal exponentials, $e^{v}$, for $v \in \Phi$ that satisfy the relations $e^{v} \cdot e^{w}=e^{v+w}$. The conjecture asserts that the constant term of $\Delta$, meaning the part that is independent of the formal exponentials, has a nice factorization as a polynomial in $q$.

Later, Macdonald [M2] generalized this work in the following way. He showed that there is a unique collection of polynomials $P_{\nu}$ indexed by dominant weights $\nu$, satisfying the following properties:

1) The $P_{\nu}$ form a basis for $\mathbf{Z}[\Phi, q]^{W}$, the $W$-invariants in $\mathbf{Z}[\Phi, q]$, where $W$ is the Weyl group of $G$. Moreover, the basis $P_{\nu}$ is triangular with respect to the basis $e_{\mu}$ of orbit sum polynomials.

2) For $\nu \neq \mu$, the constant term of

$$
\Delta \cdot P_{\nu} \cdot \hat{P}_{\mu}
$$

is zero, where $\hat{P}_{\mu}$ is obtained from $P_{\mu}$ by replacing each $e^{v}$ by $e^{-v}$.

Work partially supported by the National Science Foundation.

The authors are grateful to the Stieltjes Institute for its support of their visit to the University of Leiden during which much of this work was done. 
3) The coefficient of $e_{\nu}$ in $P_{\nu}$ is 1.

Macdonald went on to conjecture a formula for the constant term of

$$
\Delta \cdot P_{\nu} \cdot \hat{P}_{\nu}
$$

for every $\nu$. Since $P_{0}$ is the constant 1 , this conjecture extended his 1983 conjecture that gave a formula for the constant term of $\Delta$.

One interesting feature of the Macdonald conjectures is that the statement of the conjectures is not by itself of great interest. The constant term of $\Delta \cdot P_{\nu}$. $\hat{P}_{\mu}$ has no particular significance outside of the context of these conjectures. The real motivation behind any study of the Macdonald conjectures is to discover some deep mathematical phenomenon that has the constant term conjectures as a consequence.

Macdonald's constant term conjectures have been proved by Cherednik [C] using the idea of shift operators pioneered by Opdam [O] together with Cherednik's powerful affine Hecke algebra machinery. This approach does indeed give an interesting interpretation to the constant term conjectures. But there have been a number of other efforts to give mathematical interpretations to the constant term of $\Delta \cdot P_{\nu} \cdot \hat{P}_{\mu}$. These other approaches have succeeded in identifying interesting mathematical phenomena that have Macdonald's constant term conjectures as consequences. But in many of these cases, the deeper mathematical facts that underlie the Macdonald conjectures have not yet been proved.

Collectively these attempts to settle the Macdonald conjectures involve a remarkable range of mathematical subjects and ideas. Different approaches lead to interesting conjectures about the homology of nilpotent Lie algebras ([F] and $[\mathrm{H}]$ ), the harmonics of a diagonal action of the symmetric groups ([GH]), and the generalized traces of Lie algebras ([K]). In addition there is the above-mentioned work of Cherednik, Opdam and Heckman that involves shift operators, operators on polynomials that are built from elements in affine Hecke algebras. This leads one naturally to speculate that there is some deep theory that will simultaneously explain these diverse attacks on the Macdonald conjectures. At the very least one would like to understand how to relate these approaches pairwise.

One motivation for this work is an effort to connect the affine Hecke algebra approach of Cherednik with the diagonal harmonic approach of Garsia and Haiman. Garsia and Haiman study the module generated by partial derivative operators on an element in a polynomial ring in two sets of variables (this is explained more fully in Sect. 3). They use the structure of the resulting bigraded module to generate something equivalent to Macdonald's polynomials $P_{\nu}$. In this work, we replace the partial differentiation operators by Dunkl operators. Dunkl operators $D_{i}(k)$ are operators on a polynomial ring that involve a parameter $k$. Moreover, $D_{i}(k)$ is a deformation of $\partial / \partial x_{i}$ 
in the sense that $D_{i}(0)=\partial / \partial x_{i}$. The Dunkl operators are also related to the fundamental operators that define a faithful representation of the affine Hecke algebras. We hope that this work will be a starting point towards understanding the connection between the shift operator proofs of the Macdonald conjectures and the Garsia-Haiman conjectures both of which give interesting interpretations of the Macdonald polynomials.

Our main conjecture gives an explicit characterization of the singular points of this deformation. An important ingredient in this conjecture is a theorem given in Sect. 2 that gives the norms of certain special polynomials in $\mathbf{C}\left[x_{1}, \ldots, x_{n}\right]$ with respect to an inner product based on the Dunkl operators. This theorem gives a significant extension of the Mehta integral.

The paper is organized as follows. The main theorem (Theorem 2.6) is proved in Sect. 2. The computational component of the proof is quite intricate and accounts for most of the section. In Sect. 3 we discuss the relevance of this result to the Garsia-Haiman conjecture. Section 3 contains our main conjecture, which characterizes the singular points in our deformation of the Garsia-Haiman module. In Sect. 4 we describe some further conjectures that are based on computer evidence.

The authors are grateful to Eric Opdam for help and advice with this work.

\section{An extension of Mehta's integral}

Fix a positive integer $n$ and let $R$ denote the ring $\mathbb{C}\left[x_{1}, \ldots, x_{n}\right]$, where the $x_{i}$ 's are commuting indeterminates. For each $i \neq j$ let $\left(x_{i}, x_{j}\right)$ denote the endomorphism of $R$ that interchanges $x_{i}$ and $x_{j}$.

Let $p(\underline{x})$ be an element in $R$. Note that

$$
\left(i d-\left(x_{i}, x_{j}\right)\right) \cdot p(\underline{x})
$$

vanishes when $x_{i}=x_{j}$, hence is divisible by $x_{i}-x_{j}$. So the operator

$$
\frac{i d-\left(x_{i}, x_{j}\right)}{x_{i}-x_{j}}
$$

maps $R$ to $R$.

Definition 2.1 Let $k$ be an element of $\mathbb{C}$. For each $i=1,2, \ldots, n$ define $X_{i}=X_{i}(k)$ to be the map from $R$ to $R$ given by

$$
X_{i}=\frac{\partial}{\partial x_{i}}+k \sum_{j \neq i} \frac{i d-\left(x_{i}, x_{j}\right)}{x_{i}-x_{j}} .
$$


It is clear that $X_{i}$ decreases degree by 1 ("degree" means total degree in the variables $x_{1}, \ldots, x_{n}$ ). A less obvious fact (see [D]) is that the operators $X_{1}, \ldots, X_{n}$ commute pairwise. So the map from $R$ to $\mathbb{C}\left[X_{1}, \ldots, X_{n}\right] \subseteq$ $\operatorname{End}(R)$ given by

$$
p\left(x_{1}, \ldots, x_{n}\right) \longrightarrow p\left(X_{1}, \ldots, X_{n}\right)
$$

is a well-defined $S_{n}$-equivariant isomorphism.

Definition 2.2 Define the inner product $\langle\rangle=,\langle,\rangle_{k}$ on $R$ by

$$
\langle p, q\rangle= \begin{cases}0 & \text { if } \operatorname{deg}(p) \neq \operatorname{deg}(q) \\ p\left(X_{1}, \ldots, X_{n}\right) \cdot q\left(x_{1}, \ldots, x_{n}\right) & \text { if } \operatorname{deg}(p)=\operatorname{deg}(q) .\end{cases}
$$

This inner product is studied in [DDO] in connection to a certain complex.

Example 2.3 Let $n=4$ and let $\Pi \in R$ be defined by

$$
\begin{aligned}
\Pi & =:\left(x_{1}-x_{2}\right)\left(x_{3}-x_{4}\right) \\
& =x_{1} x_{3}-x_{1} x_{4}-x_{2} x_{3}+x_{2} x_{4} .
\end{aligned}
$$

We will compute $\langle\Pi, \Pi\rangle$.

First note that the inner product \langle\rangle is invariant with respect to the action of $S_{4}$ on $R$. So

$$
\begin{aligned}
\langle\Pi, \Pi\rangle & =4\left(X_{1} X_{3} \cdot\left(x_{1} x_{3}-x_{2} x_{3}-x_{1} x_{4}+x_{2} x_{4}\right)\right) \\
& =4 X_{1}\left(\left(x_{1}-x_{2}\right)+k\left(2 x_{1}-2 x_{2}+\left(x_{1}+x_{4}\right)-\left(x_{2}+x_{4}\right)\right)\right) \\
& =4(1+3 k) X_{1}\left(x_{1}-x_{2}\right) \\
& =4(1+3 k)(1+4 k) .
\end{aligned}
$$

The main result in this section will give the values of the inner products $\left\langle\Pi_{\lambda}, \Pi_{\lambda}\right\rangle$ for certain elements $\Pi_{\lambda}$ in $R$. We begin by defining the $\Pi_{\lambda}$. In what follows, $\Delta\left(u_{1}, \ldots, u_{n}\right)$ denotes $\prod_{i<j}\left(u_{i}-u_{j}\right)$.

Definition 2.4 Let $\lambda=\left(\lambda_{1}, \ldots, \lambda_{\ell}\right)$ be a partition of $n$. Define $\Pi_{\lambda}=$ $\Pi_{\lambda}\left(x_{1}, \ldots, x_{n}\right)$ by

$$
\begin{aligned}
\Pi_{\lambda}= & \Delta\left(x_{1}, \ldots, x_{\lambda_{1}}\right) \Delta\left(x_{\lambda_{1}+1}, \ldots, x_{\lambda_{1}+\lambda_{2}}\right) \\
& \cdots \Delta\left(x_{\lambda_{1}+\cdots+\lambda_{\ell-1}+1}, \ldots, x_{n}\right) .
\end{aligned}
$$

Note that $\left\langle\Pi_{\lambda}, \Pi_{\lambda}\right\rangle$ is a polynomial in $k$ of degree $\sum_{i}\left(\begin{array}{c}\lambda_{i} \\ 2\end{array}\right):=D_{\lambda}$. We will identify the $D_{\lambda}$ roots (as a polynomial in $k$ ) of $\left\langle\Pi_{\lambda}, \Pi_{\lambda}\right\rangle$ in terms of a certain sequence of numbers $\left(a_{2}^{\lambda}, \ldots, a_{n}^{\lambda}\right)$. 
Definition 2.5 Define the sequence $\left(a_{2}^{\lambda}, \ldots, a_{n}^{\lambda}\right)$ according to the following rule:

$$
\sum_{i=2}^{n} a_{i}^{\lambda} x^{i}=\sum_{j=1}^{\ell}\left(\sum_{u=1}^{\lambda_{j}-1}\left(\lambda_{j}-u\right) x^{n-u+1}\right) .
$$

As an example, let $\lambda=431^{n-7}$. Then $\left(a_{2}^{\lambda}, \ldots, a_{n}^{\lambda}\right)$ is determined by

$$
\begin{aligned}
\sum_{i=2}^{n} a_{i}^{\lambda} x^{i} & =\left(x^{n-2}+2 x^{n-1}+3 x^{n}\right)+\left(x^{n-1}+2 x^{n}\right) \\
& =x^{n-2}+3 x^{n-1}+5 x^{n}
\end{aligned}
$$

So

$$
\begin{aligned}
a_{n}^{\lambda} & =5 \\
a_{n-1}^{\lambda} & =3 \\
a_{n-2}^{\lambda} & =1 \\
a_{i}^{\lambda} & =0 \quad \text { for } 2 \leq i \leq n-3 .
\end{aligned}
$$

Note that $\sum_{i=2}^{n} a_{i}^{\lambda}=\sum_{j=1}^{\lambda}\left(\sum_{u=1}^{\lambda_{j}-1}\left(\lambda_{j}-u\right)\right)=\sum_{j=1}^{\ell}\left(\begin{array}{c}\lambda_{j} \\ 2\end{array}\right)$. Our main theorem in this section will be the following:

Theorem 2.6 Let $\lambda=\left(\lambda_{1}, \ldots, \lambda_{\ell}\right)$ be a partition of $n$ and let $H_{\lambda}$ denote $\prod_{i=1}^{\ell}\left(\lambda_{i} !\right)$. Then

$$
\left\langle\Pi_{\lambda}, \Pi_{\lambda}\right\rangle=H_{\lambda}\left\{\prod_{u=1}^{n-1} \prod_{i=(n+1)-a_{n+1-u}^{\lambda}}^{n}(i k+u)\right\}
$$

where the inside product over $u$ is empty if $a_{i}^{\lambda}=0$.

The proof of Theorem 2.6 is very complicated, and so before starting we preview the various steps it involves. First it should be noted that we will reduce the computation to the case where $\lambda$ is a hook. This reduction is essentially done in Theorem 2.30. Lemmas 2.28 and 2.29 are used in the proof of Theorem 2.30.

Before doing the reduction we prove Theorem 2.6 for $\lambda$ a hook shape $\lambda=(N+1) 1^{n-(N+1)}$. Our strategy is to reduce the computation to the same computation for $\hat{\lambda}=N 1^{n-N}$ and use induction. To do this reduction, it is necessary to compute $X_{1}^{N} \cdot \Pi_{\lambda}(\underline{x})$, which we argue is a multiple of $\Pi_{\lambda}(\underline{x})$. We need to determine what multiple. To do so, it becomes necessary to determine $X_{1}^{s} \Pi_{\lambda}(\underline{x})$ for all $s$ and most of our effort goes into this computation. 
The first step is to realize that $X_{1}^{s} \cdot \Pi_{\lambda}(\underline{x})$ is a polynomial in the differences $x_{1}-x_{i}, i=2,3, \ldots, n$. These differences play distinct roles in the computation depending on whether $2 \leq i \leq N+1$ or $N+2 \leq i \leq n$. We give them different names $\beta_{i}=x_{1}-x_{i+1}$ for $1 \leq i \leq N$ and $\alpha_{i}=$ $x_{1}-x_{N+1+i}$ for $1 \leq i \leq n-N-1$. We rewrite $X_{1}$ as an operator on $\mathbb{C}[\underline{\alpha}, \beta]$ and compute it in terms of these new variables. These preliminary computations are done in Lemmas 2.7 and 2.8.

Lemma 2.7 Let $u_{1}, \ldots, u_{d}$ be commuting indeterminates. Then

$$
\sum_{i=1}^{d} u_{i}^{s} \prod_{j \neq i} \frac{u_{j}}{u_{j}-u_{i}}= \begin{cases}1 & \text { if } s=0 \\ 0 & \text { if } 1 \leq s \leq d-1 .\end{cases}
$$

Proof. Multiply the sum on the left-hand side by $\Delta\left(u_{1}, \ldots, u_{d}\right)$. The result is a skew-symmetric polynomial in $u_{1}, \ldots, u_{d}$ of degree $s+\left(\begin{array}{l}d \\ 2\end{array}\right)$.

If $s=0$ this polynomial must be a constant multiple of $\Delta\left(u_{1}, \ldots, u_{d}\right)$, i.e., the sum on the left-hand side in the statement of Lemma 2.7 must be a constant $K$. To see that $K=1$ set $u_{i}=i-1$.

If $1 \leq s \leq d-1$ the polynomial

$$
\Delta\left(u_{1}, \ldots, u_{d}\right)\left(\sum_{i=1}^{d} u_{i}^{s} \prod_{j \neq i} \frac{u_{j}}{u_{j}-u_{i}}\right)=: Q
$$

is clearly divisible by $\left(\prod_{i=1}^{d} u_{i}\right)=: P$. Dividing $Q$ by $P$ yields a skewsymmetric polynomial $u_{1}, \ldots, u_{d}$ of degree $\left(\begin{array}{l}d \\ 2\end{array}\right)-(d-s)$, which must be 0 . So $Q=0$ and this proves Lemma 2.7.

For the next lemma we will assume that $\alpha_{1}, \ldots, \alpha_{m}, \beta_{1}, \ldots, \beta_{N}$ are commuting indeterminates. We will let $e_{r}\left(z_{1}, \ldots, z_{\ell}\right)$ denote the $r$ th elementary symmetric function in $z_{1}, \ldots, z_{\ell}$, we will let $\partial$ be the differential operator on $R=\mathbb{C}\left[\alpha_{1}, \ldots, \alpha_{m}, \beta_{1}, \ldots, \beta_{N}\right]$ given by

$$
\partial=\sum_{i=1}^{m} \frac{\partial}{\partial \alpha_{i}}+\sum_{j=1}^{N} \frac{\partial}{\partial \beta_{j}}
$$

and we will let $A_{i}, B_{j}$ be the algebra homomorphisms on $R$ that satisfy

$$
\begin{aligned}
& A_{i}(z)= \begin{cases}-\alpha_{i} & \text { if } z=\alpha_{i} \\
z-\alpha_{i} & \text { if } z \in\left\{\alpha_{1}, \ldots, \alpha_{m}, \beta_{1}, \ldots, \beta_{N}\right\} \backslash\left\{\alpha_{i}\right\}\end{cases} \\
& B_{i}(z)= \begin{cases}-\beta_{i} & \text { if } z=\beta_{i} \\
z-\beta_{i} & \text { if } z \in\left\{\alpha_{1}, \ldots, \alpha_{m}, \beta_{1}, \ldots, \beta_{N}\right\} \backslash\left\{\beta_{i}\right\} .\end{cases}
\end{aligned}
$$


Define $\gamma_{1}$ and $\gamma_{2}$ by

$$
\begin{aligned}
\gamma_{1} & =\sum_{i=1}^{m} \frac{i d-A_{i}}{\alpha_{i}} \\
\gamma_{2} & =\sum_{i=1}^{N} \frac{1}{\beta_{i}}\left[i d-\prod_{\substack{j=1 \\
j \neq i}}^{N} \frac{\beta_{j}}{\beta_{j}-\beta_{i}} B_{i}\right] .
\end{aligned}
$$

Lemma 2.8 Let $p(\underline{\alpha})$ be a polynomial in $\left(\alpha_{1}, \ldots, \alpha_{m}\right)$ of degree less than or equal to $I$. Then

$$
\begin{aligned}
\gamma_{2}\left(p(\underline{\alpha}) e_{N-I}(\underline{\beta})\right)= & \left(\sum_{i=1}^{m} \frac{\partial}{\partial \alpha_{i}}+\sum_{j=1}^{N} \frac{\partial}{\partial \beta_{i}}\right)\left(p(\underline{\alpha}) e_{N-I}(\underline{\beta})\right) \\
& +p(\underline{\alpha}) e_{N-I-1}(\underline{\beta}) .
\end{aligned}
$$

Proof. For each $j=1,2, \ldots, N$

$$
\begin{aligned}
B_{j}(p(\underline{\alpha}))=p\left(\underline{\alpha}-\beta_{j}\right) & =p\left(\alpha_{1}-\beta_{j}, \alpha_{2}-\beta_{j}, \ldots, \alpha_{m}-\beta_{j}\right) \\
& =p(\underline{\alpha})+\sum_{s=1}^{I} q_{s}(\underline{\alpha}) \beta_{j}^{s}
\end{aligned}
$$

where $q_{s}(\underline{\alpha})$ is a polynomial in $\alpha_{1}, \ldots, \alpha_{m}$. Note that the sum on the right has upper limit $s=I$ by our degree assumption on $p$.

$$
\begin{aligned}
& \gamma_{2}\left(p(\underline{\alpha}) e_{N-I}(\underline{\beta})\right)= \\
& \sum_{j=1}^{N} \frac{1}{\beta_{j}}\left(p(\underline{\alpha}) e_{N-I}(\underline{\beta})-p\left(\underline{\alpha}-\beta_{j}\right) \prod_{\ell \neq j} \frac{\beta_{\ell}}{\beta_{\ell}-\beta_{j}} B_{j}\left(e_{N-I}(\underline{\beta})\right)\right) .
\end{aligned}
$$

Expand $e_{N-I}(\beta)=\sum_{A} \beta_{A}$, where the sum is over all $(N-I)$-subsets $A$ of $\{1, \ldots, N\}$ and where $\beta_{A}$ denotes $\prod_{r \in A} \beta_{r}$.

Fix $A$ and write $\gamma_{2}\left(p(\underline{\alpha}) \beta_{A}\right)$ as a sum of two terms $G_{1}+G_{2}$ (see (2.2)) where

$$
\left.\begin{array}{rl}
G_{1} & =\sum_{j \notin A} \frac{1}{\beta_{j}}\left(p(\underline{\alpha}) \beta_{A}-p\left(\underline{\alpha}-\beta_{j}\right) \prod_{\ell \neq j} \frac{\beta_{\ell}}{\beta_{\ell}-\beta_{j}} B_{j}\left(\beta_{A}\right)\right) \\
G_{2} & =\sum_{j \in A} \frac{1}{\beta_{j}}\left(p(\underline{\alpha}) \beta_{A}-p\left(\underline{\alpha}-\beta_{j}\right) \prod_{\ell \neq j} \frac{\beta_{\ell}}{\beta_{\ell}-\beta_{j}} B_{j}\left(\beta_{A}\right)\right) .
\end{array}\right\}
$$


We can write $G_{1}$ as

$$
\begin{aligned}
G_{1}= & \beta_{A}\left(\sum_{j \notin A} \frac{1}{\beta_{j}}\left(p(\underline{\alpha})-\left(p(\underline{\alpha})+\sum_{s=1}^{I} q_{s}(\underline{\alpha}) \beta_{j}^{s}\right) \prod_{\substack{\ell \notin A \\
\ell \neq j}} \frac{\beta_{\ell}}{\beta_{\ell}-\beta_{j}}\right)\right) \\
= & \beta_{A} p(\underline{\alpha})\left(\sum_{j \notin A} \frac{1}{\beta_{j}}\left(1-\prod_{\substack{\ell \notin A \\
\ell \neq j}} \frac{\beta_{\ell}}{\beta_{\ell}-\beta_{j}}\right)\right) \\
& -\beta_{A} \sum_{s=1}^{I}\left(\sum_{j \notin A} \beta_{j}^{s-1}-\prod_{\substack{\ell \notin A \\
\ell \neq j}} \frac{\beta_{\ell}}{\beta_{\ell}-\beta_{j}}\right) q_{s}(\underline{\alpha}) .
\end{aligned}
$$

We will apply Lemma 2.7 to the second summation with $\left\{u_{1}, \ldots, u_{d}\right\}=$ $\left\{\beta_{j}: j \notin A\right\}$. Note that $d=I$ in this case whereas the highest exponent appearing in the $\beta_{j}$ is $I-1$. Lemma 2.7 gives

$$
G_{1}=\beta_{A} p(\underline{\alpha})\left(\sum_{j \notin A} \frac{1}{\beta_{j}}\left(1-\prod_{\substack{\ell \notin A \\ \ell \neq j}} \frac{\beta_{\ell}}{\beta_{\ell}-\beta_{j}}\right)\right)-\beta_{A} q_{1}(\underline{\alpha}) .
$$

Next consider $\sum_{j \notin A} \frac{1}{\beta_{j}}\left(1-\prod_{\substack{\ell \notin A \\ \ell \neq j}} \frac{\beta_{\ell}}{\beta_{\ell}-\beta_{j}}\right):=G_{3}$ and keep the notation $u_{1}, \ldots, u_{d}$ as above. Observe that

$$
\begin{aligned}
& \Delta\left(u_{1}, \ldots, u_{d}\right) G_{3}= \\
& \left(\prod_{\substack{r<s \\
r, s \notin A}}\left(\beta_{r}-\beta_{s}\right)\right)\left(\sum_{j \notin A} \frac{\left(\prod_{\substack{\ell \notin A \\
\ell \neq j}}\left(\beta_{\ell}-\beta_{j}\right)\right)-\left(\prod_{\substack{\ell \notin A \\
\ell=j}} \beta_{\ell}\right)}{\beta_{j} \prod_{\substack{\ell \notin A \\
\ell \neq j}}\left(\beta_{\ell}-\beta_{j}\right)}\right),
\end{aligned}
$$

which is a polynomial. The crucial point is that $\beta_{j}$ divides the numerator of the $j$ th summand of the right-hand factor.

But this implies that $\Delta\left(u_{1}, \ldots, u_{d}\right) G_{3}$ is 0 since it is a skew-symmetric polynomial of degree $\left(\begin{array}{l}d \\ 2\end{array}\right)-1$ in $u_{1}, \ldots, u_{d}$. So we have shown that

$$
G_{1}=-\beta_{A} q_{1}(\underline{\alpha})=-\beta_{A} \sum_{j} \frac{\partial}{\partial \alpha_{j}} p(\underline{\alpha}) .
$$


Next we consider the summand $G_{2}$ from (2.3). Expanding $B_{j}\left(\beta_{A}\right)$ according to the definition of $B_{j}$ we obtain

$$
\begin{aligned}
G_{2}= & \sum_{j \in A} \frac{1}{\beta_{j}}\left[p(\underline{\alpha}) \beta_{A}-p\left(\underline{\alpha}-\beta_{j}\right)\left(\prod_{\ell \neq j} \frac{\beta_{\ell}}{\beta_{\ell}-\beta_{j}}\right)\right. \\
& \left.\times\left(\left(-\beta_{j}\right) \prod_{\substack{r \in A \\
r \neq j}}\left(\beta_{r}-\beta_{j}\right)\right)\right] \\
= & \sum_{j \in A} \beta_{A \backslash\{j\}}\left[p(\underline{\alpha})+p\left(\underline{\alpha}-\beta_{j}\right) \prod_{\ell \notin A} \frac{\beta_{\ell}}{\beta_{\ell}-\beta_{j}}\right] .
\end{aligned}
$$

Let $B$ be a subset of $\left\{\beta_{1}, \ldots, \beta_{N}\right\}$ of size $N-I-1$. We collect together all terms in $\gamma_{2}\left(p(\underline{\alpha}) e_{N-I}(\underline{\beta})\right)$ that contribute to (2.4) with leading factor $\beta_{B}$. There is a contribution for every superset $A$ of $B$ of size $N-I$ (for such $A$ we have $\{j\}=A \backslash B)$. The coefficient of $\beta_{B}$ when we sum all such terms is

$$
\sum_{j \notin B}\left(p(\underline{\alpha})+p\left(\underline{\alpha}-\beta_{j}\right) \prod_{\substack{\ell \notin B \\ \ell \neq j}} \frac{\beta_{\ell}}{\beta_{\ell}-\beta_{j}}\right) .
$$

As above write $p\left(\underline{\alpha}-\beta_{j}\right)=p(\underline{\alpha})+\sum_{s=1}^{I} q_{s}(\underline{\alpha}) \beta_{j}^{s}$. Applying Lemma 2.7 with $\left\{u_{1}, \ldots, u_{d}\right\}=\left\{\beta_{\ell}: \ell \notin B\right\}$ we find that the collection of terms involving a $q_{s}(\alpha)$ sum to 0 . As a result, (2.5) is equal to

$$
(I+1) p(\underline{\alpha})+p(\underline{\alpha})\left(\sum_{j \notin B} \prod_{\substack{\ell \in A \\ \ell \neq j}} \frac{\beta_{\ell}}{\beta_{\ell}-\beta_{j}}\right)=(I+2) p(\underline{\alpha}) .
$$

Summing over $B$ we find that the total contribution to $\gamma_{2}\left(p(\underline{\alpha}) e_{N-I}(\underline{\beta})\right)$ from the $G_{2}$ is

$$
\begin{aligned}
(I+2) p(\underline{\alpha}) e_{N-I-1}(\underline{\beta})= & p(\underline{\alpha})\left(\left(\sum_{j=1}^{N} \frac{\partial}{\partial \beta_{j}}\right) e_{N-I}(\underline{\beta})\right) \\
& +p(\underline{\alpha}) e_{N-I-1}(\underline{\beta}) .
\end{aligned}
$$

Collecting together all terms of types $G_{1}$ and $G_{2}$ we have the result stated in Lemma 2.8.

We are constructing a proof of Theorem 2.6 that will proceed by induction. One crucial step in this proof will be the evaluation of

$$
X_{1}^{\lambda_{1}-1} \cdot \Pi_{\lambda}
$$


where $\lambda$ is written in weakly descending order $\lambda_{1} \geq \cdots \geq \lambda_{\ell}$. An important ingredient in our computation of $X_{1}^{\lambda_{1}-1} \cdot \Pi_{\lambda}$ will be an explicit formula for

$$
\left(\partial+k \gamma_{1}+k \gamma_{2}\right)^{s} \cdot e_{N}\left(\beta_{1}, \ldots, \beta_{N}\right)
$$

for all values of $s$. Lemma 2.8 is pertinent to the computation of these quantities.

Let $V$ be the vector subspace of $R$ spanned by all $p(\underline{\alpha}) e_{N-I}(\beta)$ such that the degree of $p$ is less than or equal to $I$. Lemma 2.8 implies that each of the polynomials in (2.5) lie in $V$. Let $\gamma:=k /(k+1)$ and $\alpha_{0}:=x_{1}$.

Definition 2.9 The linear operator $T$ on $V$ is defined by

$$
\begin{aligned}
T\left(p(\underline{\alpha}) e_{j}(\underline{\beta})\right): & =\sum_{i=0}^{m} \frac{\partial}{\partial \alpha_{i}} p(\underline{\alpha}) e_{j}(\underline{\beta}) \\
& +(N-j+1+\gamma) p(\underline{\alpha}) e_{j-1}(\underline{\beta})+\gamma \sum_{i=1}^{m} \frac{1-A_{i}}{\alpha_{i}} p(\underline{\alpha}) e_{j}(\underline{\beta})
\end{aligned}
$$

with $\operatorname{deg}(p)+j \leq N$.

Proposition 2.10 The operator $\partial+k \gamma_{1}+k \gamma_{2}$ equals $k /(k+1) T$ on $V$.

Proof. Combine the result of Lemma 2.8 with the fact

$$
\sum_{i=1}^{N} \frac{\partial}{\partial \beta_{i}} e_{N-I}(\underline{\beta})=(I+1) e_{N-I-1}(\underline{\beta})
$$

Proposition 2.11 The set of polynomials $\left\{p_{s}(\underline{\alpha}): s=0,1,2, \ldots\right\}$ defined by

$$
H(\rho, \underline{\alpha}):=\left(1-\rho x_{1}\right)^{-\gamma-1} \prod_{j=1}^{m}\left(1-\rho\left(x_{1}-\alpha_{j}\right)\right)^{-\gamma}=\sum_{s=0}^{\infty} p_{s}(\underline{\alpha}) \rho^{s}
$$

satisfies

$$
T\left(p_{s}(\underline{\alpha}) e_{0}\right)=((m+1) \gamma+s) p_{s-1}(\underline{\alpha}) e_{0}
$$

for all $s$ (using $e_{0}=1$ to show this is in $V$ ).

Proof. Apply $T$ to the generating function $H$. We will show that

$$
T H=\rho\left(\rho \frac{\partial}{\partial \rho}+((m+1) \gamma+1)\right) H
$$


Indeed

$$
\begin{aligned}
T H(\rho, \underline{\alpha})= & H(\rho, \underline{\alpha})\left(\frac{\rho(\gamma+1) x_{1}}{1-\rho x_{1}}\right) \\
+ & \gamma \sum_{i=1}^{m} \frac{1}{\alpha_{i}}\left\{\frac{1}{1-\rho x_{1}}-\frac{1}{1-\rho\left(x_{1}-\alpha_{i}\right)}\right\} \\
& \times\left(1-\rho x_{1}\right)^{-\gamma} \prod_{j=1}^{m}\left(1-\rho\left(x_{1}-\alpha_{j}\right)\right)^{-\gamma} \\
= & H(\rho, \underline{\alpha}) \rho\left(\frac{\gamma+1}{1-\rho x_{1}}+\gamma \sum_{i=1}^{m} \frac{1}{1-\rho\left(x_{1}-\alpha_{i}\right)}\right)
\end{aligned}
$$

while

$$
\begin{aligned}
\rho( & \left(\rho \frac{\partial}{\partial \rho}+((m+1) \gamma+1)\right) H(\rho, \underline{\alpha}) \\
= & H(\rho, \underline{\alpha})\left\{\frac{x_{1} \rho^{2}(\gamma+1)}{1-\rho x_{1}}+\gamma \rho^{2} \sum_{i=1}^{m} \frac{\left(x_{1}-\alpha_{i}\right)}{1-\rho\left(x_{1}-\alpha_{i}\right)}\right. \\
& +((m+1) \gamma+1) \rho\} \\
= & T H(\rho, \underline{\alpha}) .
\end{aligned}
$$

Proposition 2.12 For any $I=0,1,2, \ldots$ the set of polynomials $\left\{q_{I, s}(\underline{\alpha})\right.$ : $s=0,1,2, \ldots\}$ defined by

$$
G(r, \underline{\alpha}, I):=\left(1+r x_{1}\right)^{-(m+1) \gamma-I-1} \prod_{j=1}^{m}\left(1+r \alpha_{j}\right)^{\gamma}=\sum_{s=0}^{\infty} q_{I, s}(\underline{\alpha}) r^{s}
$$

satisfies $A_{l} \psi_{j}=\psi_{j}$ for $1 \leq l \leq m$ and $T \psi_{j}=0$, for $j=0, \ldots, N$; where $\psi_{j}:=\sum_{i=0}^{j} q_{N-j, i}(\underline{\alpha}) e_{j-i}(\underline{\beta})$.

Proof. Fix $j$, let $I=N-j$, then

$$
\begin{aligned}
A_{l} \psi & =\sum_{i=0}^{j} A_{l} q_{I, i}(\underline{\alpha}) e_{j-i}\left(\underline{\beta}-\alpha_{l}\right) \\
& \left.=\sum_{i=0}^{j}\left(A_{l} q_{I, i}\right)(\underline{\alpha}) \sum_{s=0}^{j-i}\left(\begin{array}{c}
s+I+i \\
s
\end{array}\right)\left(-\alpha_{l}\right)^{s} e_{j-i-s}(\underline{\beta})\right) \\
& =\sum_{t=0}^{j} e_{j-t}(\underline{\beta}) \sum_{s=0}^{t}\left(A_{l} q_{I, t-s}\right)(\underline{\alpha})\left(\begin{array}{c}
I+t \\
s
\end{array}\right)\left(-\alpha_{l}\right)^{s}
\end{aligned}
$$


where we change the variable of summation $t=i+s$ and we write $e_{j-1}(\underline{\beta-}$ $\alpha_{l}$ ) for the elementary symmetric function in the variables $\beta_{1}-\alpha_{l}, \ldots, \beta_{N}-$ $\alpha_{l}$, which is expanded in terms of $e_{j-i-s}(\beta)$ using the generating function $\sum_{i=0}^{N} e_{i}(\beta) r^{i}=\prod_{i=1}^{N}\left(1+r \beta_{i}\right)$. On the other hand, the coefficient of $r^{t}$ in the expansion of

$$
\begin{aligned}
G & \left(\frac{r}{1+r \alpha_{l}}, A_{l} \alpha, I\right)\left(1+r \alpha_{l}\right)^{-1-I} \\
& =\sum_{i=0}^{\infty}\left(A_{l} q_{I, i}\right)(\underline{\alpha}) r^{i}\left(1+r \alpha_{l}\right)^{-1-i-I} \\
& =\sum_{i=0}^{\infty}\left(A_{l} q_{I, i}\right)(\underline{\alpha}) r^{i} \sum_{s=0}^{\infty} \frac{(1+i+I)_{s}}{s !}\left(-\alpha_{l}\right)^{s} r^{s} \\
& =\sum_{t=0}^{\infty} r^{t} \sum_{s=0}^{t}\left(A_{l} q_{I, t-s}\right)(\underline{\alpha})\left(\begin{array}{c}
I+t \\
s
\end{array}\right)\left(-\alpha_{l}\right)^{s}
\end{aligned}
$$

is the coefficient of $e_{j-t}(\underline{\beta})$ in $A_{l} \psi_{j}$. But

$$
\begin{aligned}
& G\left(\frac{r}{1+r \alpha_{l}}, A_{l} \underline{\alpha}, I\right)\left(1+r \alpha_{l}\right)^{-1-I} \\
= & \left(1+\frac{r\left(x_{1}-\alpha_{l}\right)}{1+r \alpha_{l}}\right)^{-(m+1) \gamma-I-1} \prod_{j=1, j \neq l}^{m}\left(1+\frac{r\left(\alpha_{j}-\alpha_{l}\right)}{1+r \alpha_{l}}\right)^{\gamma} \\
& \times\left(1+\frac{-r \alpha_{l}}{1+r \alpha_{l}}\right)^{\gamma}\left(1+r \alpha_{l}\right)^{-I-1} \\
= & \left(1+r x_{1}\right)^{-(m+1) \gamma-I-1}\left(1+r \alpha_{l}\right)^{(m+1) \gamma+I+1-m \gamma-I-1} \\
& \times \prod_{j=1, j \neq l}^{m}\left(1+r \alpha_{j}\right)^{\gamma} \\
= & G(r, \underline{\alpha}, I)
\end{aligned}
$$

and this shows that $A_{l} \psi_{j}=\psi_{j}$. 
To show that $T \psi_{j}=0$ it suffices (because of the $A_{l}$-invariance) to evaluate

$$
\begin{aligned}
\sum_{i=0}^{j}\left\{\left[\frac{\partial}{\partial x_{1}} q_{N-j, i}(\underline{\alpha})\right] e_{j-i}(\underline{\beta})\right. & \\
+ & \left.(N-j+i+1+\gamma) q_{N-j, i}(\underline{\alpha}) e_{j-i-1}(\underline{\beta})\right\} \\
= & \sum_{i=0}^{j}\left\{\frac{\partial}{\partial x_{1}} q_{I, i}(\underline{\alpha})+(I+i+\gamma) q_{I, i-1}(\underline{\alpha})\right\} e_{j-i}(\underline{\beta})
\end{aligned}
$$

where again $I=N-j$. We need to show this is zero for $1 \leq i \leq j$; when $i=0$ this follows from $q_{I, 0}=1$. For $i \geq 1$ the expression in \{\} is the coefficient of $r^{i}$ in

$$
\begin{aligned}
& \frac{\partial}{\partial x_{1}} G(r, \underline{\alpha}, I)+r\left(I+\gamma+1+r \frac{\partial}{\partial r}\right) G(r, \underline{\alpha}, I) \\
& =G(r, \underline{\alpha}, I)\left\{-\frac{\left(r+r^{2} x_{1}\right)((m+1) \gamma+I+1)}{1+r x_{1}}\right. \\
& \left.\quad+\gamma \sum_{j+1}^{m} \frac{r+r^{2} \alpha_{j}}{1+r \alpha_{j}}+r(I+\gamma+1)\right\} \\
& =G(r, \underline{\alpha}, I) r\{-(m+1) \gamma-I-1+m \gamma+I+\gamma+1\}=0 .
\end{aligned}
$$

Corollary 2.13 For $0 \leq j \leq N$ and for any polynomial $P(\alpha)$ with $\operatorname{deg}(P)$ $\leq N-j$,

$$
T\left(P(\underline{\alpha}) \psi_{j}\right)=T(P(\underline{\alpha})) \psi_{j} .
$$

Proof. The operator $T$ has differentiation and difference components. The standard product rule applies to the differentiation, while the difference action factors through multiplication by $\psi_{j}$ because $\psi_{j}$ is invariant under each $A_{l}$.

This Corollary together with the polynomials constructed in Proposition 2.11 are essentially a diagonalization of the operator $T$ and lead to the desired formula for $T^{N} e_{N}(\beta)$. The reader will suspect that these constructions did not spontaneously jump into the authors' minds. They are the result of a series of various approaches to the problem that eventually led to the generating function method. The function $G$ arose from trying to set up a correspondence between $r^{i}$ in $G$ and $e_{j-i}(\underline{\beta})$ that exhibits the action of the 
reflection $A_{l}$. Thus $A_{l} r^{i}$ should correspond to

$$
\begin{aligned}
\sum_{s=0}^{\infty}\left(\begin{array}{c}
s+I+i \\
s
\end{array}\right)\left(-\alpha_{l}\right)^{s} r^{i+s} & =\sum_{s=0}^{\infty} \frac{(1+I+i)_{s}}{s !}\left(-\alpha_{l}\right)^{s} r^{i+s} \\
& =r^{i}\left(1+r \alpha_{l}\right)^{-1-I-i} \\
& =\left(\frac{r}{1+r \alpha_{l}}\right)^{i}\left(1+r \alpha_{l}\right)^{-1-I}
\end{aligned}
$$

(formally summing to $\infty$ results in terms like $e_{t}(\beta)$ with negative $t$; these are taken to be 0 ) and this explains the technique used in the proof of Proposition 2.12. To finish this computation we find the expansion of $e_{n}(\beta)$ in terms of $\left\{p_{s}(\underline{\alpha})\right\}$ and $\left\{\psi_{j}\right\}$.

Theorem $2.14 e_{N}(\underline{\beta})=\sum_{j=0}^{N} p_{N-j}(\underline{\alpha}) \psi_{j}$.

Proof. Start with the right side of the formula:

$$
\begin{aligned}
\sum_{j=0}^{N} p_{N-j}(\underline{\alpha}) \psi_{j} & =\sum_{j=0}^{N} \sum_{i=0}^{j} p_{N-j}(\underline{\alpha}) q_{N-j, i}(\underline{\alpha}) e_{j-i}(\underline{\beta}) \\
& =\sum_{t=0}^{N} e_{N-t}(\underline{\beta}) \sum_{s=0}^{t} p_{s}(\underline{\alpha}) q_{s, t-s}(\underline{\alpha}),
\end{aligned}
$$

(changing summation variables $i=t-s, j=N-s$ ). The coefficient of $e_{N-t}(\underline{\beta})$ in this sum equals the coefficient of $r^{t}$ in the sum

$$
\begin{aligned}
& \sum_{s=0}^{\infty} p_{s}(\underline{\alpha}) r^{s} \sum_{j=0}^{\infty} q_{s, j}(\underline{\alpha}) r^{j}=\sum_{s=0}^{\infty} p_{s}(\underline{\alpha}) r^{s} G(r, \underline{\alpha}, s) \\
& =\sum_{s=0}^{\infty} p_{s}(\underline{\alpha}) r^{s}\left(1+r x_{1}\right)^{-(m+1) \gamma-s-1} \prod_{j=1}^{m}\left(1+r \alpha_{j}\right)^{\gamma} \\
& =H\left(\frac{r}{1+r x_{1}}, \underline{\alpha}\right)\left(1+r x_{1}\right)^{-(m+1) \gamma-1} \prod_{j=1}^{m}\left(1+r \alpha_{j}\right)^{\gamma} \\
& =\left(1-\frac{r x_{1}}{1+r x_{1}}\right)^{-\gamma-1} \prod_{j=1}^{m}\left(1-\frac{r\left(x_{1}-\alpha_{j}\right)}{1+r x_{1}}\right)^{-\gamma}\left(1+r x_{1}\right)^{-(m+1) \gamma-1} \\
& \quad \times \prod_{j=1}^{m}\left(1+r \alpha_{j}\right)^{\gamma} \\
& =\left(1+r x_{1}\right)^{\gamma+1+m \gamma-(m+1) \gamma-1}=1
\end{aligned}
$$

and this establishes the desired formula. 
Corollary 2.15 For $0 \leq j \leq N$ the following formula holds:

$$
T^{j} e_{N}(\underline{\beta})=\sum_{i=0}^{N-j}((m+1) \gamma+N-i-j+1)_{j} p_{N-j-i}(\underline{\alpha}) \psi_{i}
$$

and in particular $T^{N} e_{N}(\underline{\beta})=((m+1) \gamma+1)_{N}$.

Proof. This is a consequence of Theorem 2.14 and Proposition 2.11 and Corollary 2.13.

Now we come to one of our main results.

Theorem 2.16 Let $m, N$ be as above. Then

$$
\left(\partial+k \gamma_{1}+k \gamma_{2}\right)^{N} e_{N}(\underline{\beta})=\prod_{i=1}^{N}((m+i+1) k+i) .
$$

Proof. Proposition 2.10 shows that the result of evaluating the left hand side is $(k /(k+1))^{N}$ times the value specified in Corollary 2.15.

We are now ready to proceed with the proof of Theorem 2.6. We begin with the case where $\lambda$ is a hook.

Theorem 2.17 (HOOK CASE) Let $\lambda=\left(N+1,1^{n-(N+1)}\right)$. Then

$$
\left\langle\Pi_{\lambda}, \Pi_{\lambda}\right\rangle=(N+1) ! \prod_{i=1}^{N} \prod_{u=1}^{N+1-i}((n+1-i) k+u)
$$

Proof. First note that

$$
\left\langle\Pi_{\lambda}, \Pi_{\lambda}\right\rangle=(N+1) ! X_{1}^{N} X_{2}^{N-1} \ldots X_{N} \cdot \Delta\left(x_{1}, \ldots, x_{N+1}\right) .
$$

So it is enough to show that

$$
X_{1}^{N} X_{2}^{N-1} \ldots X_{N} \cdot \Delta\left(x_{1}, \ldots, x_{N+1}\right)=\prod_{i=1}^{N=1} \prod_{u=1}^{N+1-i}((n+1-i) k+u) .
$$

We will prove (2.6) by induction on $N$. The base case $N=1$ is easy so assume that $N$ is greater than 1 and that (2.6) is known for $N-1$.

For each $s, X_{1}^{s} \cdot \Delta\left(x_{1}, \ldots, x_{N+1}\right)$ is antisymmetric in $x_{2}, \ldots, x_{N+1}$, hence divisible by $\Delta\left(x_{2}, \ldots, x_{N+1}\right)$. For each $s$, define $c_{*}=c_{s}\left(x_{1}, \ldots, x_{n}\right)$ by

$$
X_{1}^{s} \cdot \Delta\left(x_{1}, \ldots, x_{N+1}\right) .
$$


Observe also that $X_{1}^{N} \Delta\left(x_{1}, \ldots, x_{N+1}\right)$ is of degree $\left(\begin{array}{c}N \\ 2\end{array}\right)$, hence $c_{N}$ must be independent of $x_{1}, \ldots, x_{n}$. So

$$
\begin{aligned}
X_{1}^{N} X_{2}^{N-1} \ldots X_{N} & =c_{N} X_{2}^{N-1} \ldots X_{N} \Delta\left(x_{2}, \ldots, x_{N+1}\right) \\
& =c_{N}\left(\prod_{i=1}^{N-2} \prod_{u=1}^{N-i-1}((n+1-i) k+u)\right)
\end{aligned}
$$

the last equality following from our induction hypothesis.

We have reduced our problem to showing that

$$
c_{N}=\prod_{i=1}^{N}((n+1-i) k+(N+1-i))
$$

We are going to examine the functions $c_{s}$.

Let $m=n-(N+1)$. For each $i=1,2, \ldots, m$ let $\alpha_{i}=x_{1}-x_{N+1+i}$ and for each $j=1,2, \ldots, N$ let $\beta_{j}=x_{1}-x_{j+1}$. Note that $c_{0}$ in terms of this new notation is $e_{N}\left(\beta_{1}, \ldots, \beta_{N}\right)$.

Claim. Written in terms of $\alpha_{1}, \ldots, \alpha_{m}, \beta_{1}, \ldots, \beta_{N}$ we have

$$
c_{s}=\left(\partial+k \gamma_{1}+k \gamma_{2}\right)^{s} \cdot e_{N}(\underline{\beta}) \text {. }
$$

Proof of Claim. By induction on $s$. The case $s=0$ was handled above. To complete the induction step we must show that

$$
X_{1}\left(c_{s} \Delta\left(x_{2}, \ldots, x_{N+1}\right)\right)=\left(\left(\partial+k \gamma_{1}+k \gamma_{2}\right) c_{s}\right) \Delta\left(x_{2}, \ldots, x_{N+1}\right)
$$

Suppose $U_{(\underline{x})}$ is a polynomial in $x_{1}, \ldots, x_{N+1}$ that can be written as a polynomial in the set $\left\{\alpha_{1}, \ldots, \alpha_{m}, \beta_{1}, \ldots, \beta_{N}\right\}$. It is straightforward to check that $X_{1} \cdot U$ is also a polynomial in $\left\{\alpha_{1}, \ldots, \alpha_{m}, \beta_{1}, \ldots, \beta_{N}\right\}$ given by

$$
\left(\partial+k \gamma_{1}+k \tilde{\gamma}_{2}\right) \cdot U
$$

where

$$
\tilde{\gamma}_{2}=\sum_{i=1}^{N} \frac{i d-B_{i}}{\beta_{i}}
$$

Also

$$
\Delta\left(x_{2}, \ldots, x_{N+1}\right)=\Delta\left(\beta_{1}, \ldots, \beta_{N}\right)
$$


and

$$
\begin{aligned}
B_{i} \Delta\left(\beta_{1}, \ldots, \beta_{N}\right) & =\left(\prod_{\substack{j=1 \\
j \neq i}}^{N} \beta_{j}\right)\left(\prod_{\substack{u<v \\
u, v \neq i, j}}\left(\beta_{u}-\beta_{v}\right)\right) \\
& =\left(\prod_{\substack{j=1 \\
j \neq i}}^{N} \frac{\beta_{j}}{\beta_{j}-\beta_{i}}\right) \Delta\left(\beta_{1}, \ldots, \beta_{N}\right) .
\end{aligned}
$$

Hence,

$$
\begin{aligned}
X_{1} & \left(c_{s} \Delta\left(x_{2}, \ldots, x_{n+1}\right)\right)=X_{1}\left(c_{s} \Delta\left(\beta_{1}, \ldots, \beta_{N}\right)\right) \\
= & \left(\partial+k \gamma_{1}+k \gamma_{2}\right)\left(c_{s} \Delta\left(\beta_{1}, \ldots, \beta_{N}\right)\right) \\
= & \left(\left(\partial+k \gamma_{1}\right) c_{s}\right) \Delta\left(\beta_{1}, \ldots, \beta_{N}\right) \\
& +\sum_{i=1}^{N} \frac{i d-B_{i}}{\beta_{i}}\left(c_{s} \Delta\left(\beta_{1}, \ldots, \beta_{N}\right)\right) \\
= & \left(\left(\left(\partial+k \gamma_{1}\right) c_{s}\right)+k \sum_{i=1}^{N} \frac{1}{\beta_{i}}\left(i d-\prod_{j \neq i} \frac{\beta_{j}}{\beta_{i}-\beta_{j}} B_{i}\right) c_{s}\right) \\
& \cdot \Delta\left(\beta_{2}, \ldots, \beta_{N}\right) \\
= & \left(\left(\partial+k \gamma_{1}+k \gamma_{2}\right) c_{s}\right) \Delta\left(\beta_{1}, \ldots, \beta_{N}\right),
\end{aligned}
$$

which proves the Claim.

To complete the proof of (2.7) we have by the Claim above:

$$
\begin{aligned}
c_{N} & =\left(\partial+k \gamma_{1}+k \gamma_{2}\right)^{N} e_{N}(\underline{\beta}) \\
& =\prod_{j=1}^{N}(((n-(N+1))+j+1) k+j) \quad \text { by Theorem } 2.16 \\
& =\prod_{i=1}^{N}((n+1-i) k+(N+1-i))
\end{aligned}
$$

This completes the proof of Theorem 2.6 in the case that $\lambda$ is a hook.

We will now use a different argument to reduce to the hook case. Let $\lambda=\lambda_{1} \lambda_{2} \ldots \lambda_{\ell} 1^{m}$ where $2 \leq \lambda_{1} \leq \lambda_{2} \leq \cdots \leq \lambda_{\ell}$. As above, note that

$$
\left\langle\Pi_{\lambda}, \Pi_{\lambda}\right\rangle=H_{\lambda}\left(X_{1}^{\lambda_{1}-1} \ldots X_{\lambda_{1}}^{0} X_{\lambda_{1}+1}^{\lambda_{2}-1} X_{\lambda_{2}+1}^{\lambda_{2}-2} \ldots\right) \Pi_{\lambda} .
$$


We must show that

$$
\begin{aligned}
& \left(X_{1}^{\lambda_{1}-1} X_{2}^{\lambda_{1}-2} \ldots X_{\lambda_{1}}^{0} X_{\lambda_{1}+1}^{\lambda_{2}-1} X_{\lambda_{1}+2}^{\lambda_{2}-2} \ldots\right) \cdot \Pi_{\lambda} \\
& \quad=\prod_{u=1}^{n-1} \prod_{i=(n+1)-a_{n+1-u}^{\lambda}}^{n}(i k+u) .
\end{aligned}
$$

We prove (2.8) by induction on $\operatorname{deg}\left(\Pi_{\lambda}\right)$. Let $\hat{\lambda}=\left(\lambda_{1}-1\right) \lambda_{2} \ldots \lambda_{\ell} 1^{m+1}$. Since

$$
\operatorname{deg}\left(X_{1}^{\lambda_{1}-1} \Pi_{\lambda}\right)=\sum\left(\begin{array}{c}
\hat{\lambda}_{i} \\
2
\end{array}\right)
$$

and since $X_{1}^{\lambda_{1}-1} \Pi_{\lambda}$ is antisymmetric in the sets

$$
\begin{aligned}
& \left\{x_{2}, \ldots, x_{\lambda_{1}}\right\} \\
& \left\{x_{\lambda_{1}+1}, \ldots, x_{\lambda_{1}+\lambda_{2}}\right\} \\
& \quad \vdots \\
& \left\{x_{\lambda_{1}+\cdots+\lambda_{\ell-1}+1}, \ldots, x_{\lambda_{1}+\cdots+\lambda_{\ell}}\right\}
\end{aligned}
$$

we have that $X_{1}^{\lambda_{1}-1} \Pi_{\lambda}$ is a constant multiple of $\Pi_{\lambda}$. Let that multiple be denoted by $C$. Our first step is to write down what we expect this multiple to be. For $\mu=\mu_{1} \ldots \mu_{\ell}$ a partition of $n$, let $R_{\mu}(k)$ be the product on the right-hand side of Theorem 2.6 (divided by $H_{\mu}$ ). More precisely, let

$$
R_{\mu}(k)=\prod_{u=1}^{n-1} \prod_{i=(n+1)-a_{n+1-u}^{\lambda}}^{n}(i k+u) .
$$

Lemma 2.28 Let $\lambda$ and $\hat{\lambda}$ be as above. Then

$$
R_{\lambda}(k) / R_{\hat{\lambda}}(k)=\prod_{i=1}^{\lambda_{1}-1}((i \ell+1) k+i) .
$$

Proof. By the definition of the $a_{i}^{\lambda}$ (Definition 2.5) we have that

$$
R_{\lambda}(k) / R_{\hat{\lambda}}(k)=\prod_{i=1}^{\lambda_{1}-1}\left(v_{i} k+i\right)
$$


where

$$
\begin{aligned}
v_{1}= & \left((n+1)-\left(\lambda_{1}-1\right)-\left(\lambda_{2}-1\right)-\cdots-\left(\lambda_{\ell}-1\right)\right)=\ell+1+m, \\
v_{2}= & \left((n+1)-\left(\lambda_{1}-2\right)-\left(\lambda_{2}-2\right)-\cdots-\left(\lambda_{\ell}-2\right)\right)=2 \ell+1+m, \\
& \vdots \\
v_{\lambda_{1}-1}= & \left((n+1)-\left(\lambda_{1}-\left(\lambda_{1}-1\right)\right)-\left(\lambda_{2}-\left(\lambda_{1}-1\right)\right)-\cdots\right. \\
& \left.-\left(\lambda_{\ell}-\left(\lambda_{1}-1\right)\right)\right) \\
= & \left(\lambda_{1}-1\right) \ell+1+m,
\end{aligned}
$$

which proves the lemma.

In view of Lemma 2.28, Theorem 2.6 will follow from a proof of

$$
X_{1}^{\lambda_{1}-1} \Pi_{\lambda}=\left(\prod_{i=1}^{\lambda_{1}-1}((i \ell+m+1) k+i)\right) \Pi_{\hat{\lambda}} .
$$

To prove (2.9) we will need one more computation.

Lemma 2.29 Suppose $1<a<b$ and suppose $0 \leq s \leq(b-a)$. Then

$$
\left(\sum_{j=a}^{b} \frac{i d-\left(x_{1}, x_{j}\right)}{x_{1}-x_{j}}\right) x_{1}^{s} \Delta\left(x_{a}, \ldots, x_{b}\right)=s x_{1}^{s-1} \Delta\left(x_{a}, \ldots, x_{b}\right)
$$

Proof. (Using Lemma 2.7) Evaluate

$$
\begin{aligned}
& \sum_{j=a}^{b} \frac{i d-\left(x_{1}, x_{j}\right)}{x_{1}-x_{j}} x_{1}^{s} \Delta\left(x_{a}, \ldots, x_{b}\right) \\
& =\sum_{j=a}^{b} \frac{1}{x_{1}-x_{j}}\left[x_{1}^{s}-x_{j}^{s} \prod_{\substack{\ell \neq j \\
a \leq \ell \leq b}} \frac{x_{1}-x_{\ell}}{x_{j}-x_{\ell}}\right] \Delta\left(x_{a}, \ldots, x_{b}\right) .
\end{aligned}
$$

Now let $u_{i}=x_{1}-x_{a-1+i}, 1 \leq i \leq b-a+1$.

It is required to find

$$
\sum_{j=1}^{b-a+1} \frac{1}{u_{j}}\left(x_{1}^{s}-\left(x_{1}-u_{j}\right)^{s} \prod_{\substack{\ell \neq j \\ 1 \leq \ell \leq b-a+1}} \frac{u_{\ell}}{u_{\ell}-u_{j}}\right)
$$

The coefficient of $x_{1}^{s}$ is

$$
\sum_{j=1}^{b-a+1} \frac{1}{u_{j}}\left(1-\prod_{\ell \neq j} \frac{u_{\ell}}{u_{\ell}-u_{j}}\right)
$$


a symmetric rational function, with no singularity at any $u_{j}$; it has degree -1 and becomes an alternating polynomial when multiplied by $\prod_{i<j}\left(u_{i}-u_{j}\right)$. But then it must be zero (by a degree argument, see a similar device in the proof of formula (2.3) in Lemma 2.8). Next, the coefficient of $x_{1}^{s-m}$ (for $1 \leq m \leq s)$ is

$$
(-1)^{m+1}\left(\begin{array}{c}
s \\
m
\end{array}\right) \sum_{j=1}^{b-a+1} u_{j}^{m-1} \prod_{\ell \neq j} \frac{u_{\ell}}{u_{\ell}-u_{j}},
$$

which equals $s$ for $m=1$, and equals 0 for $1 \leq m-1 \leq b-a$ (by Lemma 2.7 ) that is, $2 \leq m \leq b-a+1$, and the largest possible value for $m$ is $s \leq b-a+1$.

An immediate corollary of Lemma 2.29 is the following result.

Theorem 2.30 Suppose $q\left(x_{1}\right)$ is any polynomial in $x_{1}$ of degree less than or equal to $\lambda_{2}$. Then

$$
\begin{gathered}
\left(\sum_{j=\lambda_{1}+1}^{\lambda_{1}+\cdots+\lambda_{\ell}} \frac{i d-\left(x_{1}, x_{j}\right)}{x_{1}-x_{j}}\right) \cdot q\left(x_{1}\right) \Delta\left(x_{\lambda_{1}+1}, \ldots, x_{\lambda_{1}+\lambda_{2}}\right) \ldots \\
\Delta\left(x_{\lambda_{1}+\cdots+\lambda_{\ell-1}+1}, \ldots, x_{\lambda_{1}+\cdots+\lambda_{\ell}}\right) \\
=\left((\ell-1) \frac{\partial}{\partial x_{1}} q\left(x_{1}\right)\right) \Delta\left(x_{\lambda_{1}+1}, \ldots, x_{\lambda_{1}+\lambda_{2}}\right) \\
\ldots \Delta\left(x_{\lambda_{1}+\cdots+\lambda_{\ell-1}+1}, \ldots, x_{\lambda_{1}+\cdots+\lambda_{\ell}}\right) .
\end{gathered}
$$

We are now ready to prove (2.9), which will complete the proof of Theorem 2.6. We will apply Theorem 2.30 repeatedly to

$$
q_{s}\left(x_{1}\right)=\mathcal{O}_{k}^{s} \Delta\left(x_{1}, \ldots, x_{\lambda_{1}}\right)
$$

where $\mathcal{O}_{k}$ is the operator

$$
\mathcal{O}_{k}=\frac{\partial}{\partial x_{1}}+k \sum_{j=2}^{\lambda_{1}} \frac{i d-\left(x_{1}, x_{j}\right)}{x_{1}-x_{j}}+k \sum_{j=\left(\sum \lambda_{i}\right)+1}^{n} \frac{i d-\left(x_{1}, x_{j}\right)}{x_{1}-x_{j}}
$$

(note that $q\left(x_{1}\right)$ involves the variables $x_{1}, \ldots, x_{\lambda_{1}}$ as well as $\left.x_{\left(\lambda_{1}+\cdots+\lambda_{\ell}\right)+1}, \ldots, x_{n}\right)$. Let $z$ denote $\Delta\left(x_{\lambda_{1}+1}, \ldots, x_{\lambda_{1}+\lambda_{2}}\right) \ldots$ $\Delta\left(x_{\lambda_{1}+\cdots+\lambda_{\ell-1}+1}, \ldots, x_{\lambda_{1}+\cdots+\lambda_{\ell}}\right)$. By Theorem 2.30 together with the observation that

$$
X_{1}=\mathcal{O}_{k}+k \sum_{j=\lambda_{1}+1}^{\lambda_{1}+\cdots+\lambda_{\ell}} \frac{i d-\left(x_{1}, x_{j}\right)}{x_{1}-x_{j}}
$$


we have

$$
\begin{aligned}
X_{1}^{s} \Pi_{\lambda} & =\left(\left\{\mathcal{O}_{k}+k(\ell-1) \frac{\partial}{\partial x_{1}}\right\}^{s} \Delta\left(x_{1}, \ldots, x_{\lambda_{1}}\right)\right) z \\
& =(1+k(\ell-1))^{s}\left(\mathcal{O}_{k /(1+k(\ell-1))}^{s} \Delta\left(x_{1}, \ldots, x_{\lambda_{1}}\right)\right) z .
\end{aligned}
$$

Note that the hook case of Theorem 2.6 (which we've already proved) applies to the computation of

$$
\mathcal{O}_{k /[1+k(\ell-1)]}^{s} \Delta\left(x_{1}, \ldots, x_{\lambda_{1}}\right)
$$

to give

$$
\begin{aligned}
X_{1}^{\lambda_{1}-1} \Pi_{\lambda}= & (1+k(\ell-1))^{\lambda_{1}-1}\left\{\prod_{j=1}^{\lambda_{1}-1}\left(\left(\lambda_{1}+m\right)+1-j\right)\right. \\
& \left.\times\left(\frac{k}{1+k(\ell-1)}\right)+\left(\lambda_{1}-j\right)\right\} \Pi_{\hat{\lambda}} \\
= & \left\{\prod_{i=1}^{\lambda_{1}-1}((m+i+1) k)+i((\ell-1) k+1)\right\} \Pi_{\hat{\lambda}} \\
= & \left\{\prod_{i=1}^{\lambda_{1}-1}((i \ell+m+1) k+i)\right\} \Pi_{\hat{\lambda}},
\end{aligned}
$$

which proves (2.9) and completes the proof of Theorem 2.6.

It is interesting to note that the inner product computed by Theorem 2.6 can be rewritten as an integral. Theorem 3.8 from Dunk1 [D2] implies that

$$
\left\langle\Pi_{\lambda}, \Pi_{\lambda}\right\rangle=c_{k} \int_{\mathbb{R}^{n}} \Pi_{\lambda}(x)^{2}\left|\Delta\left(x_{1}, \ldots, x_{n}\right)\right|^{2 k} e^{-|x|^{2} / 2} d x
$$

where

$$
c_{k}:=\left(\int_{\mathbb{R}^{n}}\left|\Delta\left(x_{1}, \ldots, x_{n}\right)\right|^{2 k} e^{-|x|^{2} / 2} d x\right)^{-1}
$$

and $k \geq 0$ (or $\operatorname{Re}(k) \geq 0$ ) because $\Pi_{\lambda}(x)$ is $k$-harmonic, that is, annihilated by $\sum_{i=1}^{n} X_{i}^{2}$. It is the polynomial of minimum degree with its alternating properties. The latter integral is known as the Macdonald-Mehta-Selberg integral. A method of evaluation that applies to all Weyl groups was found by Opdam [O]. The results of the present paper give an independent approach. Indeed

$$
c_{k}^{-1}=(2 \pi)^{-n / 2} \prod_{j=2}^{n}\left(\frac{\Gamma(j k+1)}{\Gamma(k+1)}\right) .
$$


We prove this for $k=1,2,3, \ldots$; the extension to complex values of $k$ with $R e(k) \geq 0$ is a standard argument based on Carlson's theorem. Applying the formula (2.11) with the choice $\lambda=(n)$, Theorem 2.6 shows that

$$
\begin{aligned}
c_{k+1}^{-1} & =c_{k}^{-1}\left(n ! \prod_{i=2}^{n} \prod_{u=1}^{i-1}(i k+u)\right) \\
& =c_{k}^{-1}\left(\prod_{i=2}^{n}\left(\prod_{u=1}^{i}(i k+u)\right) /(k+1)\right) .
\end{aligned}
$$

Clearly $c_{0}^{-1}=(2 \pi)^{-n / 2}$ and for $k=0,1,2,3, \ldots$ this formula shows

$$
c_{k}^{-1}=c_{0}^{-1} \prod_{i=2}^{n}\left(\frac{(i k) !}{k !}\right) \text {. }
$$

\section{The Garsia-Haiman Conjecture}

In this section $\left\{x_{1}, \ldots, x_{n}, y_{1}, \ldots, y_{n}\right\}$ will be two sets of commuting indeterminates and $R$ will be $\mathbb{C}\left[x_{1}, \ldots, x_{n}, y_{1}, \ldots, y_{n}\right]$.

Definition 3.1 Let $k_{x}$ and $k_{y}$ be complex numbers. For each $i=1,2, \ldots, n$ define $X_{i}=X_{i}\left(k_{x}\right)$ and $Y_{i}=Y_{i}\left(k_{y}\right)$ by

$$
\begin{aligned}
& X_{i}=\frac{\partial}{\partial x_{i}}+k_{x} \sum_{j \neq i} \frac{i d-\left(x_{i}, x_{j}\right)}{x_{i}-x_{j}} \\
& Y_{i}=\frac{\partial}{\partial y_{i}}+k_{y} \sum_{j \neq i} \frac{i d-\left(y_{i}, y_{j}\right)}{y_{i}-y_{j}}
\end{aligned}
$$

The Garsia-Haiman conjecture concerns the $S_{n}$-module structure of the subspace of $R$ spanned by all partial derivatives of all orders applied to a certain starting vector $v_{\lambda}(\underline{x}, \underline{y})$.

Definition 3.2 Let $\lambda$ be a partition ofn. Let $t_{\lambda}$ be the Young tableau obtained by filling the Ferrer's diagram of $\lambda$ with the numbers $1,2, \ldots, n$ starting along the first row from left to right, then the second row from left to right, etc. For each $i$, let $r_{i}$ and $c_{i}$ be the row and column of the square in $t_{\lambda}$ containing $i$.

Define $m_{\lambda}=m_{\lambda}(\underline{x}, \underline{y})$ to be the monomial

$$
m_{\lambda}=\prod_{i=1}^{n} x_{i}^{c_{i}-1} y_{i}^{r_{i}-1}
$$


Lastly define $u_{\lambda}=u_{\lambda}(\underline{x}, \underline{y})$ by

$$
u_{\lambda}=\sum_{\sigma \in S_{n}} \operatorname{sgn}(\sigma) \sigma \cdot m_{\lambda}
$$

where $\sigma \cdot m_{\lambda}$ is the monomial obtained from $m_{\lambda}$ by replacing each $x_{i}$ with $x_{\sigma^{-1} i}$ and each $y_{i}$ with $y_{\sigma^{-1} i}$.

Example 3.3 Let $\lambda=31$ so $n=4$. Then $t_{\lambda}$ is given by

$$
t_{\lambda}=\begin{aligned}
& 123 \\
& 4
\end{aligned}
$$

A chart of $r_{i}, c_{i}$ appears below:

$$
\begin{array}{ccccc}
i & 1 & 2 & 3 & 4 \\
r_{i} & 1 & 1 & 1 & 2 \\
c_{i} & 1 & 2 & 3 & 1
\end{array}
$$

So

$$
m_{\lambda}=x_{1}^{0} y_{1}^{0} x_{2}^{1} y_{2}^{0} x_{3}^{2} y_{3}^{0} x_{4}^{0} y_{4}^{1}=x_{2} x_{3}^{2} y_{4} .
$$

The polynomial $v_{\lambda}$ is a sum of 24 distinct terms:

$$
u_{\lambda}=x_{2} x_{3}^{2} y_{4}-x_{1} x_{3}^{2} y_{4}-x_{3} x_{2}^{2} y_{4}-\cdots .
$$

By construction, $\sigma \cdot u_{\lambda}=\operatorname{sgn}(\sigma) u_{\lambda}$ for $\sigma \in S_{n}$. So the one-dimensional subspace spanned by $u_{\lambda}$ is $S_{n}$-invariant.

Definition 3.4 Let $\lambda$ be a partition of $n$ and let $k_{x}$ and $k_{y}$ be complex numbers. Define $\mathcal{V}_{\lambda}\left(k_{x}, k_{y}\right)$ to be the subspace of $R$ spanned by all $X_{i_{1}} X_{i_{2}} \ldots$ $X_{i_{r}} Y_{j_{1}} Y_{j_{2}} \ldots Y_{j_{s}} \cdot v_{\lambda}$ for $r, s$ non-negative and $i_{1}, \ldots, i_{r}, j_{1}, \ldots, j_{s}$ $\in\{1,2, \ldots, n\}$.

Note that $\mathcal{V}_{\lambda}\left(k_{x}, k_{y}\right)$ is invariant under the diagonal action of $S_{n}$ on $R$. Hence $\mathcal{V}_{\lambda}\left(k_{x}, k_{y}\right)$ has the structure of an $S_{n}$-module. We begin with the following conjecture due to Garsia and Haiman (see [G], [GH1]-[GH5] for a complete development of their work):

Conjecture 3.5 (see [GH1]) For every partition $\lambda$ of $n, \mathcal{V}_{\lambda}(0,0)$ is isomorphic to the regular representation of $S_{n}$.

In this section we are going to discuss the deformations $\mathcal{V}_{\lambda}\left(k_{x}, k_{y}\right)$ of the $S_{n}$-module $\mathcal{V}_{\lambda}(0,0)$. Straightforward arguments from linear algebra imply that the $S_{n}$-module structure of $\mathcal{V}_{\lambda}\left(k_{x}, k_{y}\right)$ is the same outside a singular set $\Omega_{\lambda}$ of pairs $\left(k_{x}, k_{y}\right)$ that has measure 0 . We will call a pair $\left(k_{x}, k_{y}\right)$ singular for $\lambda$ if $\left(k_{x}, k_{y}\right)$ is in $\Omega_{\lambda}$. Otherwise we will say that $\left(k_{x}, k_{y}\right)$ is generic for $\lambda$. 
Example 3.6 Let $\lambda=21$. In this case we have

$$
u_{\lambda}=x_{2} y_{3}-x_{1} y_{3}-x_{3} y_{2}-x_{2} y_{1}+x_{3} y_{1}+x_{1} y_{2} \text {. }
$$

It is straightforward to check that

$$
\Omega_{21}=\left\{\left(-\frac{1}{3}, k_{y}\right): k_{y} \in \mathbb{C}\right\} \cup\left\{\left(k_{x},-\frac{1}{3}\right): k_{x} \in \mathbb{C}\right\} .
$$

An interesting problem is to determine the exact set of pairs $\left(k_{x}, k_{y}\right)$ in the singular set $\Omega_{\lambda}$. The following conjecture, if true, gives a solution to this problem.

For $p(\underline{x}, \underline{y}), q(\underline{x}, \underline{y}) \in R$, we let $\langle p, q\rangle$ denote

$$
\langle p, q\rangle=\left\{\begin{array}{l}
0 \text { if the total degrees of } p \text { and } q \text { are different } \\
p\left(X_{1}, \ldots, X_{n}, Y_{1}, \ldots, Y_{n}\right) \cdot q\left(x_{1}, \ldots, x_{n}, y_{1}, \ldots, y_{n}\right) \\
\quad \text { if } \operatorname{deg}(p)=\operatorname{deg}(q) .
\end{array}\right.
$$

This pairing $\langle$,$\rangle is the two-variable analogue of the inner product introduced$ in Sect. 2.

Conjecture 3.7 Let $\lambda$ be a partition of $n$. Then $\left(k_{x}, k_{y}\right)$ is singular for $\lambda$ if and only if

$$
\left\langle v_{\lambda}(\underline{x}, \underline{y}), v_{\lambda}(\underline{x}, \underline{y})\right\rangle=0 .
$$

We have a significant amount of computational evidence in favor of this conjecture. We will discuss this data in the next section along with refinements of Conjecture 3.7. Note the similarity in statement between Conjecture 3.7 and the results in Sect. 4 of [DDO].

Return now to Example 3.6. Computing $\left\langle v_{\lambda}(\underline{x}, \underline{y}), v_{\lambda}(\underline{x}, \underline{y})\right\rangle$ we have

$$
v_{\lambda}(\underline{X}, \underline{Y}) \cdot v_{\lambda}(\underline{x}, \underline{y})=6\left(X_{2} Y_{3} \cdot v_{\lambda}(\underline{x}, \underline{y})\right) .
$$

Using the fact that

$$
X_{i}\left(x_{j}\right)= \begin{cases}1+(n-1) k_{x} & \text { if } i=j \\ -k_{x} & \text { if } i \neq j\end{cases}
$$

(and similarly for $Y_{i}\left(y_{j}\right)$ ) we have

$$
\begin{aligned}
v_{\lambda}(\underline{X}, \underline{Y}) \cdot v_{\lambda}(\underline{x}, \underline{y})= & 6\left(\left(1+2 k_{x}\right)\left(1+2 k_{y}\right)+k_{x}\left(1+2 k_{y}\right)\right. \\
& \left.-k_{x} k_{y}+\left(1+2 k_{x}\right) k_{y}+k_{x} k_{y}+k_{x} k_{y}\right) \\
= & 6\left(1+3 k_{x}\right)\left(1+3 k_{y}\right) .
\end{aligned}
$$

So $\left\langle v_{\lambda}(\underline{x}, \underline{y}), v_{\lambda}(\underline{x}, \underline{y})\right\rangle=0$ if and only if one of $k_{x}$ or $k_{y}$ is equal to $-\frac{1}{3}$. This is exactly the set $\Omega_{\lambda}$ computed in Example 3.6.

In the example above $\left\langle v_{\lambda}(\underline{x}, \underline{y}), v_{\lambda}(\underline{x}, \underline{y})\right\rangle$ factored as a polynomial in $k_{x}$ times a polynomial in $k_{y}$. The next theorem gives a factorization of $\left\langle v_{\lambda}(\underline{x}, \underline{y}), v_{\lambda}(\underline{x}, \underline{y})\right\rangle$ for all $\lambda$. 
Theorem 3.8 Let $\lambda$ be a partition of $n$ and let $\lambda^{\prime}$ denote the conjugate partition. Then

$$
\left\langle v_{\lambda}(\underline{x}, \underline{y}), v_{\lambda}(\underline{x}, \underline{y})\right\rangle=\Lambda\left\langle\Pi_{\lambda}(\underline{x}), \Pi_{\lambda}(\underline{x})\right\rangle\left\langle\Pi_{\lambda^{\prime}}(\underline{y}), \Pi_{\lambda^{\prime}}(\underline{y})\right\rangle
$$

where $\Lambda$ is the constant

$$
\Lambda=\left(H_{\lambda} H_{\lambda^{\prime}} \prod_{i=2}^{n} a_{i}^{\lambda} ! a_{i}^{\lambda^{\prime}} !\right) / n ! \prod_{i=1}^{n}\left(r_{i}-1\right) !\left(c_{i}-1\right) ! .
$$

and where the $a_{i}$ are as defined in Definition 2.5.

Before proceeding with the proof it should be pointed out that $\left\langle\Pi_{\lambda}(\underline{x}), \Pi_{\lambda}(\underline{x})\right\rangle$ is a polynomial in $k_{x}$ whose factorization was determined explicitly in Theorem 2.6 and $\left\langle\Pi_{\lambda^{\prime}}(\underline{y}), \Pi_{\lambda^{\prime}}(y)\right\rangle$ is a corresponding polynomial in $k_{y}$.

Proof. We begin with some general remarks. Let $\mu$ be a partition of $n$ and let $M_{\mu}$ be the linear span in $\mathbb{C}\left[z_{1}, \ldots, z_{n}\right]$ of the $\operatorname{set}\left\{\sigma \cdot \Pi_{\mu}\left(z_{1}, \ldots, z_{n}\right)\right.$ : $\left.\sigma \in S_{n}\right\}$. By construction $M_{\mu}$ is an $S_{n}$-module. A theorem of Peel (see [P]) asserts that $M_{\mu}$ is isomorphic to the irreducible representation $\tau_{\mu}$ of $S_{n}$ indexed by $\mu$.

Let $f_{\mu}$ denote the degree of $\tau_{\mu}$, which is known to be the number of standard Young tableaux (SYT's) of shape $\mu$. For each standard Young tableau $t$ let $\sigma_{t}$ denote the permutation in $S_{n}$ satisfying

$$
\sigma_{t}\left(t_{\mu}\right)=t
$$

( $t_{\mu}$ was defined in Definition 3.2). It is known that a basis for $M_{\mu}$ is given by the set of

$$
\left\{\sigma_{t}\left(\Pi_{\mu}\right): t \text { is an SYT of shape } \mu\right\} \text {. }
$$

Let this basis be denoted by $\left\{p_{1}^{\prime}, \ldots, p_{f_{\mu}}^{\prime}\right\}$.

Suppose now that $\langle p, q\rangle$ is the $S_{n}$-invariantinner product on $\mathbb{C}\left[z_{1}, \ldots, z_{n}\right]$ given by $\langle p, q\rangle=p\left(Z_{1}, \ldots, Z_{n}\right) \cdot q\left(z_{1}, \ldots, z_{n}\right)$ where

$$
Z_{i}=\frac{\partial}{\partial z_{i}}+k \sum_{j \neq i} \frac{i d-\left(z_{i}, z_{j}\right)}{z_{i}-z_{j}} .
$$

With respect to this inner product $\left\{p_{1}^{\prime}, p_{2}^{\prime}, \ldots\right\}$ is not generally orthogonal. By use of the Gram-Schmidt method this basis can be replaced by an orthogonal basis $\left\{p_{1}, p_{2}, p_{3}, \ldots\right\}$ such that $p_{1}^{\prime}=p_{1}$, and the matrix representation of the group $S_{n}$ is orthogonal. This of course simultaneously implies that the $p_{i}$ 's all have the same norm. 
Lemma 3.9 If $i \neq j$ then $\left\langle p_{i}, p_{j}\right\rangle=0$. The value of $\left\langle p_{i}, p_{i}\right\rangle$ is independent of $i$.

Proof. For $\sigma \in S_{n}$ we have $\sigma\left(p_{i}\right)=\sum_{s}\left(\tau_{\mu}(\sigma)\right)_{s, i} p_{s}$ and we have $\left\langle p_{i}, p_{j}\right\rangle=$ $\left\langle\sigma p_{i}, \sigma p_{j}\right\rangle$. Combining these yields,

$$
\begin{aligned}
\left\langle p_{i}, p_{j}\right\rangle & =\frac{1}{n !} \sum_{\sigma \in S_{n}}\left\langle\sigma p_{i}, \sigma p_{j}\right\rangle \\
& =\sum_{r, s}\left(\frac{1}{n !} \sum_{\sigma \in S_{n}}\left(\tau_{\mu}(\sigma)\right)_{r, i}\left(\tau_{\mu}(\sigma)\right)_{s, j}\right)\left\langle p_{r}, p_{s}\right\rangle .
\end{aligned}
$$

It is a well-known fact from the representation theory of finite groups (see $[\mathrm{F}])$ that

$$
\frac{f_{\mu}}{n !} \sum_{\sigma \in S_{n}}\left(\tau_{\mu}(\sigma)\right)_{r, i}\left(\tau_{\mu}(\sigma)\right)_{s, j}=\delta_{r, s} \delta_{i j}
$$

(here we use that $\tau_{\mu}$ is real). Thus

$$
\left\langle p_{i}, p_{j}\right\rangle=\frac{\delta_{i j}}{f_{\mu}} \sum_{s}\left\langle p_{s}, p_{s}\right\rangle .
$$

Let $M$ be the subspace of $\mathbb{C}\left[x_{1}, \ldots, x_{n}\right]$ described above that affords the representation $\tau_{\lambda}$ and let $M^{\prime}$ be the corresponding subspace of $\mathbb{C}\left[y_{1}, \ldots, y_{n}\right]$ that affords the representation $\tau_{\lambda^{\prime}}$. Let $p_{1}(\underline{x}), \ldots, p_{f_{\mu}}(\underline{x})$ be the basis described above for $M$ and let $q_{1}(\underline{y}), \ldots, q_{f_{\mu}}(\underline{y})$ be the corresponding basis for $M^{\prime}$ that satisfies

$$
\sigma\left(q_{i}(\underline{y})\right)=\sum_{s}\left(\tau_{\lambda}(\sigma)\right)_{s, i} \operatorname{sgn}(\sigma) q_{s}(\underline{y}) .
$$

We are going to consider $M \otimes M^{\prime}$ as a subspace of $\mathbb{C}\left[x_{1}, \ldots, x_{n}, y_{1}, \ldots, y_{n}\right]$. The $S_{n}$ action on $\mathbb{C}\left[x_{1}, \ldots, x_{n}, y_{1}, \ldots, y_{n}\right]$ will be by simultaneous permutation of the $x_{i}$ 's and $y_{j}$ 's so that $M \otimes M^{\prime}$, as an $S_{n}$-module, is isomorphic to the internal tensor product of $\tau_{\lambda}$ and $\tau_{\lambda^{\prime}}$. It follows from standard facts about the representation theory of $S_{n}$ that $M \otimes M^{\prime}$ contains exactly one copy of the sign representation. The next lemma identifies that element and computes its norm squared.

Lemma 3.10 In the tensor product $M \otimes M^{\prime}$ there is a unique polynomial (up to scalar multiple) that is skew-symmetric. This polynomial is given by

$$
v=\sum_{i=1}^{f_{\lambda}} p_{i}(\underline{x}) q_{i}(\underline{y}) .
$$

Moreover $\langle v, v\rangle=f_{\lambda}\left\langle p_{1}, p_{1}\right\rangle\left\langle q_{1}, q_{1}\right\rangle$. 
Proof. Let $\sigma \in S_{n}$. Then

$$
\begin{aligned}
\sigma v & =\operatorname{sgn}(\sigma) \sum_{r, s} p_{r}(\underline{x}) q_{s}(\underline{y})\left(\sum_{i}\left(\tau_{\lambda}(\sigma)\right)_{r, i}\left(\tau_{\lambda}(\sigma)\right)_{s, i}\right) \\
& =\operatorname{sgn}(\sigma) \sum_{r, s} p_{r}(\underline{x}) q_{s}(\underline{y}) \delta_{r s}
\end{aligned}
$$

here using the orthogonality relation

$$
\left(\sum_{i}\left(\tau_{\lambda}(\sigma)\right)_{r, i}\left(\tau_{\lambda}(\sigma)\right)_{s, i}\right)=\delta_{r s}
$$

(see $[\mathrm{F}])$. So

$$
\sigma \cdot v=\operatorname{sgn}(\sigma) v
$$

which shows that the linear span of $v$ is the unique copy of the sign representation in $M \otimes M^{\prime}$. Note that

$$
\begin{aligned}
\langle v, v\rangle & =\left\langle\sum_{i} p_{i}(\underline{x}) q_{i}(\underline{y}), \sum_{j} p_{j}(\underline{x}) q_{j}(\underline{y})\right\rangle \\
& =\sum_{i, j}\left\langle p_{i}(\underline{x}), p_{j}(\underline{x})\right\rangle\left\langle q_{i}(\underline{y}), q_{j}(\underline{y})\right\rangle \\
& =\sum_{i}\left\langle p_{i}(\underline{x}), p_{i}(\underline{x})\right\rangle\left\langle q_{i}(\underline{y}), q_{i}(\underline{y})\right\rangle \\
& =f_{\lambda}\left\langle p_{1}(\underline{x}), p_{1}(\underline{x})\right\rangle\left\langle q_{1}(\underline{y}), q_{1}(\underline{y})\right\rangle
\end{aligned}
$$

(the last two equalities following from Lemma 3.9). This proves Lemma 3.10 .

To prove Theorem 3.8 we are going to apply this set-up to $v_{\lambda}(\underline{x}, \underline{y})$. We need one last ingredient.

Lemma 3.11 Let notation be as above. Then $v_{\lambda}(\underline{x}, \underline{y}) \in M \otimes M^{\prime}$.

Proof. Let $D_{\lambda}$ and $D_{\lambda^{\prime}}$ be defined by

$$
\begin{aligned}
D_{\lambda} & =\sum_{i}\left(\begin{array}{c}
\lambda_{i} \\
2
\end{array}\right) \\
D_{\lambda^{\prime}} & =\sum_{j}\left(\begin{array}{c}
\lambda_{j}^{\prime} \\
2
\end{array}\right) .
\end{aligned}
$$


Let $A$ be the subspace of $\mathbb{C}\left[x_{1}, \ldots, x_{n}\right]$ consisting of polynomials of degree $D_{\lambda}$ and let $B$ be the subspace of $\mathbb{C}\left[y_{1}, \ldots, y_{n}\right]$ consisting of polynomials of degree $D_{\lambda^{\prime}}$.

Consider the coefficient in $v_{\lambda}(\underline{x}, \underline{y})$ of the monomial $\prod_{i} y_{i}^{r_{i}-1}$ (this coefficient is a polynomial in $x_{1}, \ldots, x_{n}$ ). It is easy to see that this coefficient is $\Pi_{\lambda}(\underline{x})$. It follows that the coefficient in $v_{\lambda}(\underline{x}, \underline{y})$ of every monomial $y_{1}^{\alpha_{1}} \ldots y_{n}^{\alpha_{n}}$ is an element of $M$. This shows that

$$
v_{\lambda}(\underline{x}, \underline{y}) \in M \otimes B .
$$

A similar argument shows that

$$
v_{\lambda}(\underline{x}, \underline{y}) \in A \otimes M^{\prime} .
$$

We can write $A=M \oplus N$ and $B=M^{\prime} \oplus N^{\prime}$. It follows that

$$
(M \otimes B) \cap\left(A \otimes M^{\prime}\right)=M \otimes M^{\prime},
$$

which proves Lemma 3.11 .

To continue with the proof of Theorem 3.8 we note that $v_{\lambda}(\underline{x}, y)$ is in the $S_{n}$-isotypic component of $M \otimes M^{\prime}$ corresponding to the sign representation. So $v_{\lambda}(\underline{x}, \underline{y})$ is some multiple of $v=\sum_{i=1}^{f} p_{i}(\underline{x}) q_{i}(\underline{y})$. By Lemma 3.10,

$$
\begin{aligned}
\left\langle v_{\lambda}(\underline{x}, \underline{y}), v_{\lambda}(\underline{x}, \underline{y})\right\rangle & =\Lambda\left\langle p_{1}, p_{1}\right\rangle\left\langle q_{1}, q_{1}\right\rangle \\
& =\Lambda\left\langle\Pi_{\lambda}(\underline{x}), \Pi_{\lambda}(\underline{x})\right\rangle\left\langle\Pi_{\lambda^{\prime}}(\underline{y}), \Pi_{\lambda^{\prime}}(\underline{y})\right\rangle
\end{aligned}
$$

where $\Lambda$ is a constant (independent of $k_{x}, k_{y}$ ).

Both sides of the equation above are polynomials in $k_{x}$ and $k_{y}$. We will evaluate $\Lambda$ by computing the constant term of both sides. The constant term is obtained by applying only the partial derivative operators of each $X_{i}$ and $Y_{j}$. Doing so we see

$$
\begin{aligned}
C T\left(\left\langle v_{\lambda}(\underline{x}, \underline{y}), v_{\lambda}(\underline{x}, \underline{y})\right\rangle\right) & =n ! \prod_{i}\left(\frac{\partial}{\partial y_{i}}\right)^{r_{i}-1}\left(\frac{\partial}{\partial x_{i}}\right)^{c_{i}-1} y_{i}^{r_{i}-1} x_{i}^{c_{i}-1} \\
& =n ! \prod_{i}\left(r_{i}-1\right) !\left(c_{i}-1\right) !
\end{aligned}
$$

On the other hand,

$$
C T\left(\left\langle\Pi_{\lambda}(\underline{x}), \Pi_{\lambda}(\underline{x})\right\rangle\right)=H_{\lambda} \prod_{i=2}^{n} a_{i}^{\lambda} ! .
$$

Theorem 3.9 follows immediately. 
Table 1.

$$
\begin{aligned}
& \lambda=3 \quad \begin{array}{|c|c|c|c|c|}
\hline a \backslash b & 3 & 2 & 1 & 0 \\
\hline 0 & & & & \\
\hline
\end{array} \\
& \lambda=21 \quad \begin{array}{c|c|c|}
\hline a \backslash b & 1 & 0 \\
\hline 1 & & \\
0 & -1 / 3 & -1 / 3 \\
\hline
\end{array}
\end{aligned}
$$

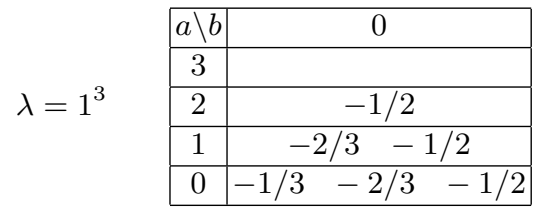

A simple corollary of Theorem 3.8 is the following:

Corollary 3.12 (a) Let $\Omega=\operatorname{set}\{-i /(j+1): j=1,2, \ldots, n-1,1 \leq i$ $\leq j\}$. Then $\left\langle v_{\lambda}(\underline{x}, \underline{y}), v_{\lambda}(\underline{x}, \underline{y})\right\rangle$ is nonzero if both $k_{x}, k_{y}$ are from $\mathbb{C} \backslash \Omega$.

(b) (Assuming CONJECTURE 3.7): The pair $(0,0)$ is generic for all $\lambda$.

\section{Refinements of Conjecture 3.7}

The $S_{n}$-module $\mathcal{V}_{\lambda}\left(k_{x}, k_{y}\right)$ is bigraded by homogeneous degree in the $x_{i}$ 's and $y_{j}$ 's. For non-negative integers $a, b$ let $V_{\lambda}^{(a, b)}\left(k_{x}, k_{y}\right)$ denote the $(a, b)$ piece of $\mathcal{V}_{\lambda}\left(k_{x}, k_{y}\right)$ that is homogeneous of degree $a$ in the $x_{i}$ 's and $b$ in the $y_{j}$ 's. We have computed the $V_{\lambda}^{(a, b)}\left(k_{x}, k_{y}\right)$ as $S_{n}$-modules for all $a, b, k_{x}$, $k_{y}$ and all $\lambda$ with $|\lambda| \leq 6$. For the sake of brevity we will not include all this data but will include certain tables that are derived from it.

The following tables are indexed by partitions $\lambda$. The $\lambda$ th table has rows indexed by $a$ for $0 \leq a \leq D_{\lambda}$ and columns indexed by $b$ for $0 \leq b \leq D_{\lambda^{\prime}}$. The $a, b$ entry in this table is a list of all $k_{y}$ such that the $(a, b)$-graded piece of $V_{\lambda}^{(a, b)}\left(k_{x}, k_{y}\right)$ is 0 for $k_{x}$ generic. (See Table 1)

Note that when $V_{\lambda}^{(a, b)}\left(k_{x}, k_{y}\right)=0$ for $k_{y}$ generic then $V_{\lambda}^{(a, i)}\left(k_{x}, k_{y}\right)=0$ for all $i$. This observation is consistent with all the data we have. This says that there is a cut-off value of $a$ for each $k_{x}$ below which $V_{\lambda}^{(a, i)}\left(k_{x}, k_{y}\right)$ is 0 (for $k_{y}$ generic).

The following value of $a$ seems to work. 
Conjecture 4.1 Suppose $k_{x}=-\frac{u}{i}$ for $u \leq a_{i}^{\lambda}$ (so in particular $\left\langle v_{\lambda}(\underline{x}, \underline{y}\right.$ ), $\left.\left.v_{\lambda}(\underline{x}, \underline{y})\right\rangle=0\right)$. Then

$$
V_{\lambda}^{(a, b)}\left(k_{x}, k_{y}\right)=0
$$

for all $b$, all $k_{y}$ and all $a \leq u+\left(\begin{array}{c}n-i+2 \\ 2\end{array}\right)-1$.

Note that the bound on $a$ is independent of $\lambda$. The significance of the independence from $\lambda$ in the previous tables is that whenever $-\frac{1}{4}$ is singular for $\lambda, V_{\lambda}^{(a, b)}\left(-\frac{1}{4}, k_{y}\right)$ was nonzero until $a=0$. Similarly whenever $\left(-\frac{1}{4}, k_{y}\right)$ is singular for $\lambda, V_{\lambda}^{(a, b)}\left(-\frac{1}{4}, k_{y}\right)$ is zero iff $a \leq 1$.

A weaker form of Conjecture 4.1 is the following:

Conjecture 4.2 The pair $\left(k_{x}, k_{y}\right)$ is singular for $\lambda$ if and only if $V_{\lambda}^{(0,0)}\left(k_{x}, k_{y}\right)=0$.

We strongly believe Conjecture 4.2 is true but we cannot as yet prove it. The following theorem implies that Conjecture 4.2 is stronger than Conjecture 3.7 .

Theorem 4.3 For all $\lambda, k_{x}$ and $k_{y}$, the following two statements are equivalent:

$$
\begin{aligned}
V_{\lambda}^{(0,0)}\left(k_{x}, k_{y}\right) & \neq 0, \\
\left\langle v_{\lambda}(\underline{x}, \underline{y}), v_{\lambda}(\underline{x}, \underline{y})\right\rangle & \neq 0 .
\end{aligned}
$$

Proof. It is immediate from the definition of $\langle$,$\rangle that (b) implies (a).$

To prove that (a) implies (b) assume that $\left\langle v_{\lambda}(\underline{x}, \underline{y}), v_{\lambda}(\underline{x}, \underline{y})\right\rangle=0$. We want to show that $V_{\lambda}^{(0,0)}\left(k_{x}, k_{y}\right)$ is 0 .

As in the proof of Lemma 3.11 we let $\left(D_{\lambda}, D_{\lambda^{\prime}}\right)$ be the bigrading of $v_{\lambda}(\underline{x}, \underline{y})$, let $A$ be the subspace of polynomials of degree $D_{\lambda}$ in $\mathbb{C}\left[x_{1}, \ldots, x_{n}\right]$ and let $B$ be the subspace of polynomials of degree $D_{\lambda^{\prime}}$ in $\mathbb{C}\left[y_{1}, \ldots, y_{n}\right]$. Let $M$ and $M^{\prime}$ be the $S_{n}$-modules in $A$ and $B$ generated by $\Pi_{\lambda}(\underline{x})$ and $\Pi_{\lambda^{\prime}}(\underline{y})$ respectively. We will need the following classical result.

Lemma 4.4 The multiplicity of the irreducible representation $\tau_{\lambda}$ in $A$ is one (and so the multiplicity of the irreducible representation $\tau_{\lambda^{\prime}}$ in $B$ is one).

Let $\partial_{X} \partial_{Y}$ be a differential operator in $\mathbb{C}\left[X_{1}, \ldots, X_{n}, Y_{1}, \ldots, Y_{n}\right]$ of bi-degree $\left(D_{\lambda}, D_{\lambda^{\prime}}\right)$ and assume that $\partial_{X}$ comes from the $\tau_{\mu}$-isotypic component of the differential operators of degree $D_{\lambda}$ whereas $\partial_{Y}$ comes from the $\tau_{\eta}$-isotypic component of the differential operators of degree $D^{\lambda^{\prime}}$.

By considering the $S_{n} \times S_{n}$ action on $A \otimes B$ we have that

$$
\left.\partial_{X} \partial_{Y}\right|_{M \otimes M^{\prime}}=0
$$


unless $\mu=\lambda$ and $\eta=\lambda^{\prime}$. By Lemma 4.4, $\partial_{X} \partial_{Y} v_{\lambda}(\underline{x}, \underline{y}) \cdot$ must come from the copy of $M \otimes M^{\prime}$ in $\mathbb{C}\left[X_{1}, \ldots, X_{n}, Y_{1}, \ldots, Y_{n}\right]$.

Consider the diagonal action of $S_{n}$ on $A \otimes B$ and on the copy of $A \otimes B$ in $\mathbb{C}\left[X_{1}, \ldots, X_{n}, Y_{1}, \ldots, Y_{n}\right]$. In order for $\partial_{X} \partial_{Y} \cdot v_{\lambda}(\underline{x}, \underline{y})$ to be nonzero, $\partial_{X} \partial_{Y}$ must come from the sign-isotypic component of this diagonal action (since $v_{\lambda}(\underline{x}, \underline{y})$ does). So by Lemma $3.10, \partial_{X} \partial_{Y}$ must be a multiple of $v_{\lambda}(\underline{X}, \underline{Y})$. But by assumption

$$
v_{\lambda}(\underline{X}, \underline{Y}) \cdot v_{\lambda}(\underline{x}, \underline{y})=0
$$

which shows that $\partial_{X} \partial_{Y} \cdot v_{\lambda}(\underline{x}, \underline{y})=0$ for all operators $\partial_{X} \partial_{Y}$. Thus $V_{\lambda}^{(0,0)}\left(k_{x}, k_{y}\right)=0$.

\section{References}

[D1] Dunkl, C. F., Differential-Difference Operators Associated to Reflection Groups. Trans. Amer. Math. Soc. 311 (1989), 167-183

[D2] Dunkl, C. F., Integral Kernals with Reflection Group Invariance. Can. J. Math. 43 (1991), 1213-1227

[DDO] Dunkl, C. F., de Jeu, M. F. E., Opdam, E., Singular Polynomials for Finite Reflection Groups. Trans. Amer. Math. Soc. 346 (1994), 237-256

[F] Feit, W., The Representation Theory of Finite Groups (1982), North-Holland Publishing Company

[G] Garsia, A., Recent Progress on the Macdonald $q, t$-Kostka Conjecture. Actes du $4^{e}$ Colloque sur les Séries Formelles et Combinatoire Algebrique, Lab. de Comb. et Informatique Mathematique, UQAM collection (1992), 249-255

[GH1] Garsia, A., Haiman, M., A GRADED Representation Model for Macdonald's Polynomials. Proc. Nat. Acad. Sci. (1993), 3607-3610

[GH2] Garsia, A., Haiman, M., Orbit Harmonics and Graded Representations. Lab. de Comb. et Informatique Mathematique, UQAM Collection, S. Brlek, Editor (to appear)

[GH3] Garsia, A., Haiman, M., Some Natural Bigraded $S_{n}$-Modules and the $q, t$-Kostka Coefficients. Electron Combin 3 (1966), \#24

[GH4] Garsia, A., Haiman, M., Factorizations of Pieri Rates for Macdonald Polynomials. Proc. of 4th Colloquium on Algebraic Combinatorics. Discrete Math. 139 (1995), 219-256

[GH5] Garsia, A., Haiman, M., A Remarkable $q, t$-Catalan Sequence and $q$-Lagrange Inversion. Journal of Algebraic Combinatorics 5 (1996), 191-244

[M] Macdonald, I. G., Symmetric Functions and Hall Polynomials (1979), Oxford University Press

[O] Opdam, E., Some Applications of Hypergeometric Shift Operators. Inv. Math. 98 (1989), 1-18

[P] Peel, M. H., Specht Modules and the Symmetric Groups. J. Alg. 36 (1975), 88-97 